\title{
RESEARCH
}

Open Access

\section{Mitochondrial respiratory chain deficiency correlates with the severity of neuropathology in sporadic Creutzfeldt- Jakob disease}

Irene H. Flønes ${ }^{1,2}$, Gerda Ricken³ ${ }^{3}$ Sigrid Klotz ${ }^{3}$, Alexandra Lang ${ }^{3}$, Thomas Ströbel ${ }^{3}$, Christian Dölle ${ }^{1,2}$, Gabor G. Kovacs ${ }^{3,4,5^{*}+}$ and Charalampos Tzoulis ${ }^{1,2^{*}+}$

\begin{abstract}
Mitochondrial dysfunction has been implicated in multiple neurodegenerative diseases but remains largely unexplored in Creutzfeldt-Jakob disease. Here, we characterize the mitochondrial respiratory chain at the individual neuron level in the MM1 and W2 common molecular subtypes of sporadic Creutzfeldt-Jakob disease. Moreover, we investigate the associations between the mitochondrial respiratory chain and neuropathological markers of the disease.

Brain tissue from individuals with sporadic Creutzfeldt-Jakob disease and age-matched controls were obtained from the brain collection of the Austrian Creutzfeldt-Jakob Surveillance. The mitochondrial respiratory chain was studied through a dichotomous approach of immunoreactivities in the temporal cortex and the hippocampal subregions of CA4 and CA3.

We show that profound deficiency of all mitochondrial respiratory complexes (I-V) occurs in neurons of the severely affected temporal cortex of patients with Creutzfeldt-Jakob disease. This deficiency correlates strongly with the severity of neuropathological changes, including vacuolation of the neuropil, gliosis and disease associated prion protein load. Respiratory chain deficiency is less pronounced in hippocampal CA4 and CA3 regions compared to the temporal cortex. In both areas respiratory chain deficiency shows a predilection for the MM1 molecular subtype of Creutzfeldt-Jakob disease.

Our findings indicate that aberrant mitochondrial respiration could be involved early in the pathogenesis of sporadic Creutzfeldt-Jakob disease and contributes to neuronal death, most likely via ATP depletion. Based on these results, we propose that the restricted MRI diffusion profile seen in the brain of patients with sporadic CreutzfeldtJakob disease might reflect cytotoxic changes due to neuronal respiratory chain failure and ATP loss.
\end{abstract}

Keywords: Mitochondria, Respiratory chain, Prion, Neurodegeneration, PrP

\footnotetext{
*Correspondence: gabor.kovacs@uhnresearch.ca; gabor.kovacs@utoronto.ca; charalampos.tzoulis@nevro.uib.no; charalampos.tzoulis@helse-bergen.no

${ }^{\dagger}$ Gabor G. Kovacs and Charalampos Tzoulis contributed equally to this work. ${ }^{3}$ Institute of Neurology, Medical University of Vienna, Vienna, Austria

${ }^{1}$ Neuro-SysMed, Department of Neurology, Haukeland University Hospital, 5021 Bergen, Norway

Full list of author information is available at the end of the article
}

C C The Author(s). 2020 Open Access This article is licensed under a Creative Commons Attribution 4.0 International License, which permits use, sharing, adaptation, distribution and reproduction in any medium or format, as long as you give appropriate credit to the original author(s) and the source, provide a link to the Creative Commons licence, and indicate if changes were made. The images or other third party material in this article are included in the article's Creative Commons licence, unless indicated otherwise in a credit line to the material. If material is not included in the article's Creative Commons licence and your intended use is not permitted by statutory regulation or exceeds the permitted use, you will need to obtain permission directly from the copyright holder. To view a copy of this licence, visit http://creativecommons.org/licenses/by/4.0/ The Creative Commons Public Domain Dedication waiver (http://creativecommons.org/publicdomain/zero/1.0/) applies to the data made available in this article, unless otherwise stated in a credit line to the data. 


\section{Introduction}

Sporadic Creutzfeldt-Jakob disease (sCJD) is a severe and lethal neurodegenerative disease that commonly manifests between the sixth and seventh decade of life with an overall incidence of 1-2/million / year [1]. While it is most often recognized by rapidly progressive dementia and myoclonus, it has a broad spectrum of clinical presentations including ataxia, visual disturbances, tremor and other movement disorders. The neuropathology of sCJD is characterized by neuronal loss, vacuolation of the neuropil, reactive gliosis and disease-associated prion protein $\left(\mathrm{PrP}^{\mathrm{sc}}\right)$ deposits [2]. $\operatorname{Pr}^{\mathrm{sc}}$ are pathological aggregations of prion protein $\left(\operatorname{PrP}^{\mathrm{c}}\right)$, a naturally occurring protein of unclear physiological function encoded by the PRNP gene. SCJD differs from other neurodegenerative proteinopathies in that self-aggregating $\operatorname{PrP}^{\mathrm{sc}}$ is considered to be the primary disease-causing event, and that it is transmissible through nerve to nerve contact [1]. $\mathrm{PrP}^{\mathrm{sc}}$ aggregation is disease specific and is found both in subtle synaptic and perineuronal accumulations and more granular, perivacuolar and plaque-like accumulations depending on disease subtype [2]. PrP ${ }^{\mathrm{sc}}$ has been shown to exist in molecular subtypes that show differences in size and degree of glycosylation, degree of protease resistance, aggregation state and conformational stability; namely $\operatorname{PrP}^{\mathrm{sc}}$ type 1 and $2[3,4]$.

Clinical and pathological features of sCJD are strongly influenced by the molecular subtype of $\operatorname{PrP} \mathrm{P}^{\mathrm{sc}}$ and the genotype of codon 129 of the PRNP gene, coding for either methionine or valine $[2,5]$. Individuals who are homozygous for the methionine codon (MM) and have type $1 \mathrm{PrP}^{\mathrm{sc}}$ (MM1) typically develop rapidly progressive dementia and myoclonus. Pathological examination commonly shows involvement of neocortex, striatum and thalamus, while the hippocampus and brain stem are relatively spared [4]. Individuals who are homozygous for the valine codon (VV) and have type $2 \mathrm{PrP}^{\mathrm{sc}}$ (VV2) often present with ataxia and develop dementia at later stages. Pathologically, individuals with VV2 show involvement of the deep neocortical layers, subcortical nuclei and the hippocampal formation [4].

Mitochondrial dysfunction, including mitochondrial respiratory chain (MRC) deficiency and mitochondrial DNA (mtDNA) damage, has been implicated in multiple neurodegenerative diseases [6], but has not been as extensively explored in SCJD. Mitochondrial abnormalities, including morphological alterations, MRC deficiency and impaired mitochondrial dynamics have been reported by studies in cell- and animal models of prion disease [7-10]. Furthermore, one study indicated decreased RNA and protein expression levels for several MRC subunits in human bulk brain tissue [11]. However, since disease-associated changes in cell-type composition have been shown to confound mitochondrial measurements in bulk brain tissue
$[12,13]$, it is unknown to what degree the reported findings reflected MRC depletion in individual cells, or altered cell composition in the CJD samples. While decreased immunofluorescent staining for subunits of complexes I, IV and $\mathrm{V}$ was also observed in SCJD [11], this has not been systematically studied. Thus, the question of whether aberrant mitochondrial function occurs within neurons of the sCJD brain remains largely unexplored.

In this work, we study the MRC complexes in single neurons of severely and mildly affected regions in the brain of individuals with either MM1 or VV2 molecular subtypes of sCJD. Our findings show that there is deficiency of all MRC complexes in the temporal cortex of sCJD. Interestingly, MRC deficiency has a predilection for the MM1 molecular subtype of sCJD and is strongly associated with neuropathological markers of the disease.

\section{Material and methods}

\section{Patient cohorts and tissue samples}

Brain tissue from individuals with $\operatorname{SCJD}(n=20)$ and controls $(n=6)$ was obtained from the brain collection of the Austrian Creutzfeldt-Jakob Surveillance at the Institute of Neurology, Medical University of Vienna. We chose to study two regions showing different pathological involvement in SCJD: (i) the temporal cortex, which is severely affected, and (ii) the CA4 and CA3 subregions of the hippocampus, which are considered relatively spared of neuropathological changes [14]. Brains were collected at autopsy and sampling was performed as previously described [15]. All samples were collected using a standard technique and a mean fixation time of 30 days. Tissue with suspected prion disease was treated in $98 \%$ formic acid (FA) for one hour prior to paraffin embedding according to routine. Our material includes three neurologically healthy controls (Ctrl), and three disease-controls $\left(\mathrm{Ctrl}_{\mathrm{D}}\right)$ in which CJD was initially suspected but excluded after neuropathological examination. $\mathrm{Ctrl}_{\mathrm{D}}$ showed signs of neurodegeneration including senile neurodystrophic changes and chronic vascular lesions, but an exact diagnosis was not available. $\mathrm{Ctrl}_{\mathrm{D}}$ were included to study the effect of FA treatment on the tissue, as neurologically healthy controls had not been FA treated. Individuals with SCJD were grouped according to codon 129 polymorphism of the PRNP gene and PrP ${ }^{\mathrm{sc}}$ type $\left(\mathrm{n}_{\mathrm{MM} 1}=10, \mathrm{n}_{\mathrm{VV} 2}=10\right)$ based on neuropathological examination as previously described [16]. All included individuals were examined by a neuropathologist at autopsy and sections were stained with hematoxylin and eosin using a standard protocol and with antibodies against $\operatorname{PrP}^{\mathrm{sc}}$ (12F10sc, SPIbio \#A03221, dilution 1: 2000). Sections were autoclaved at $121^{\circ} \mathrm{C}$ for $10 \mathrm{~min}$ following $5 \mathrm{~min}$ treatment in concentrated FA and proteinase K. All sections were stained using the Autostainer Link 48 by Agilent Dako. There was no difference in 
post-mortem delay, formalin fixation time or age between controls, and individuals with MM1- or VV2 molecular subtypes of sCJD. Furthermore, there was no difference in the disease duration between individuals in the MM1 and VV2 groups. Sex was not balanced between the controls ( 5 females and 1 male) and patients (9 females and 11 males). As previous studies have not shown gender-dependent differences in mitochondrial staining [17], we deemed this acceptable.

\section{Immunohistochemistry}

Immunohistochemistry (IHC) for mitochondrial markers was carried out on formalin-fixed, paraffin embedded sections from the hippocampus and temporal cortex of individuals with $\mathrm{SCJD}$ and controls $\left(\mathrm{Ctrl}_{\mathrm{D}}\right.$ and $\left.\mathrm{Ctrl}\right)$. Serial sections with a thickness of $3 \mu \mathrm{m}$ were deparaffinised in xylene and rehydrated in graded ethanol. Antigen retrieval was performed in $1 \mathrm{mM}$ EDTA at $\mathrm{pH} 8$. All sections were stained using Autostainer Link 48 by Agilent Dako. Sections were blocked in peroxidase-blocking solution. Primary antibodies (all from Abcam) against MRC complexes; complex I (anti-NDUFB8, ab110242, dilution 1: 4000), complex II (anti-SDHA, ab14715, dilution 1: 10,000), complex III (anti-UQCRC2, ab14745, dilution 1: 20,000), complex IV (anti-MTCO1, ab14705, dilution 1: 20,000), complex V (anti-ATP5A, ab14748, dilution 1: 20,000), and the mitochondrial membrane marker VDAC1 (anti-VDAC1, ab14734, dilution 1: 20, 000) were diluted in EnVision ${ }^{\text {TM }}$ FLEX Antibody Diluent and were incubated at room temperature for $30 \mathrm{~min}$. HRP (horseradish peroxidase)-polymer and DAB (3,3'Diaminobenzidine) chromogen kits were used for visualization. Peroxidase-blocking solution, TBST buffer, mouse-linker, HRP-polymer and DAB chromogen were all from Agilent (EnVision FLEX+, Mouse, High $\mathrm{pH}$ (Link), K8002).

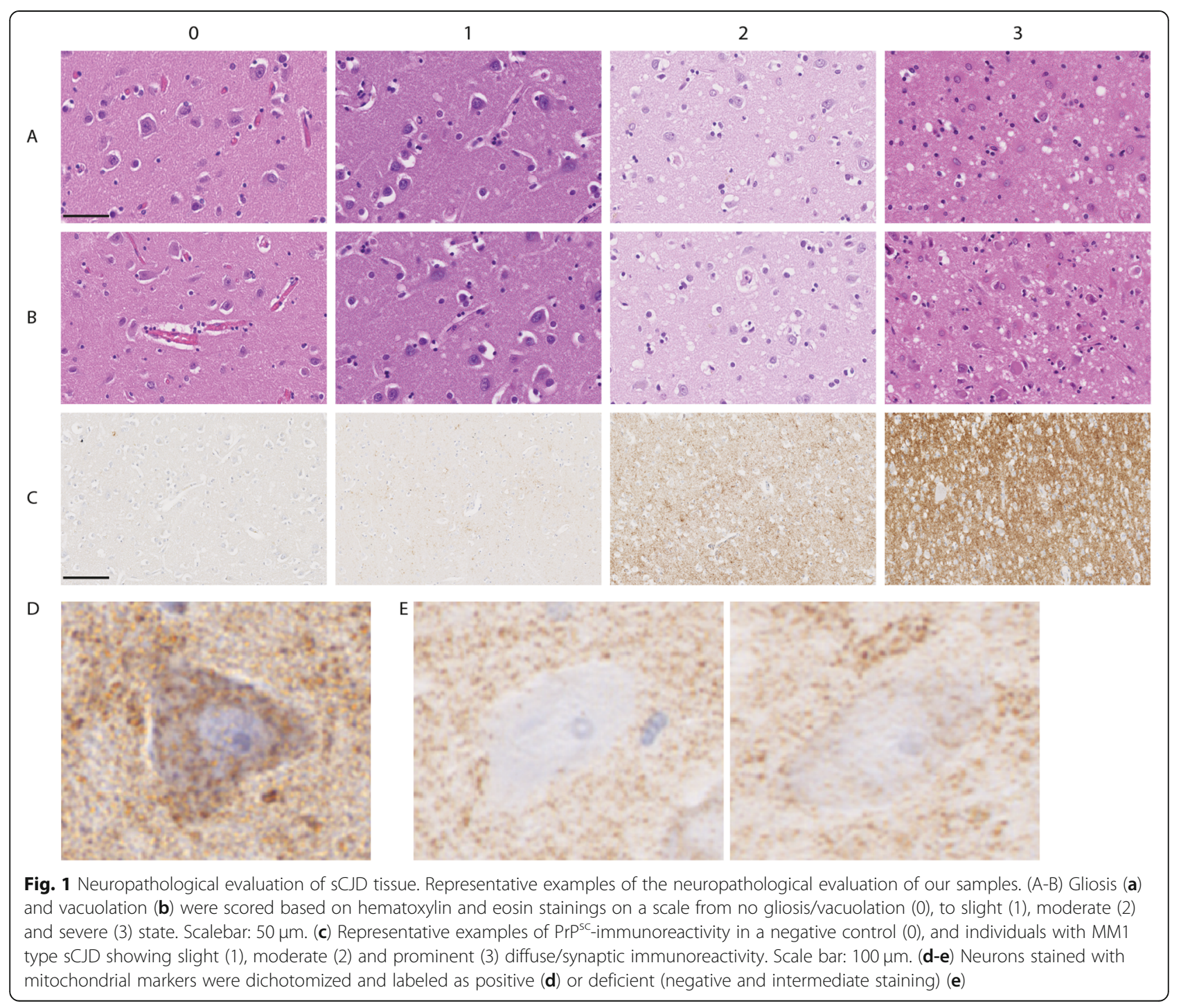




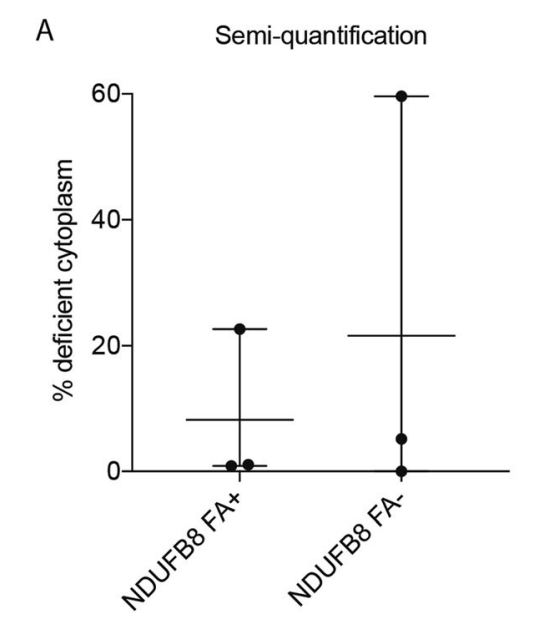

B

Visual analyses

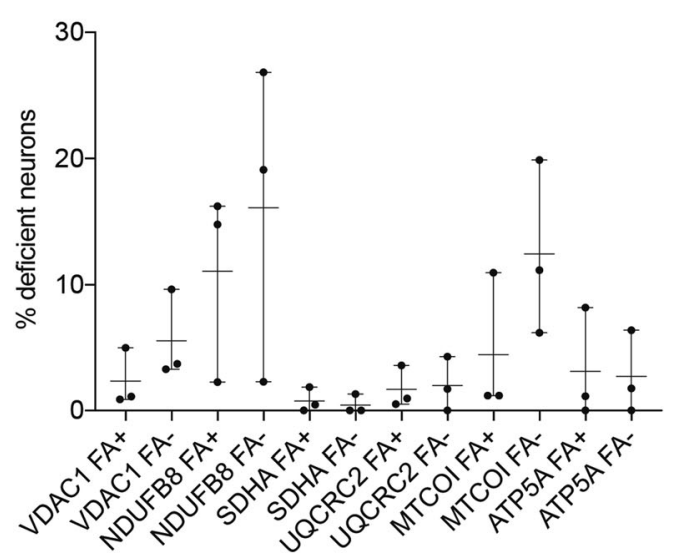

Fig. 2 Effect of formic acid treatment on immunohistochemistry. Formic acid treated (FA+) and untreated (FA-) tissue from disease-controls was compared using two different approaches. Findings from analyses in the temporal cortex are shown. a Semi-quantification of neuronal cytoplasmic stain, here exemplified by complex I (NDUFB8), shows less MRC deficiency in FA+ compared to FA- from the same three individuals. b Visual analysis and categorization of the same sections and individuals resulted in more consistent results. \% deficient cytoplasm: mean percentage of neuronal unstained cytoplasm, \% deficient neurons: percentage of total neurons from $2.5 \mathrm{~mm}^{2}$ of temporal cortex that did not stain positive. Bars show mean and range

\section{Immunohistochemistry analysis}

Both FA treated and -untreated tissue was available from the $\mathrm{Ctrl}_{\mathrm{D}}$-group, which allowed us to study the effect of FA pretreatment in single individuals. Only the FA treated sections were later included for comparisons between SCID and the entire control group. To evaluate the effect of FA treatment, tissue from the $\mathrm{Ctrl}_{\mathrm{D}}$-group that had either been FA treated or not was analysed using a semi-quantitative method to calculate the fraction of positive pixels per neuron as previously described [17]. All sections stained with antibodies against MRC complexes or VDAC1 were analyzed visually and the percentage of positive or deficient neurons was determined in the entire CA4, including the transition into $\mathrm{CA} 3$, and in a $2.5 \mathrm{~mm}^{2}$ area in the temporal cortex in close relation to the transition from entorhinal cortex into the temporal cortex. Neurons were identified based on morphology, and only neurons where the nucleus and nucleolus were clearly visible were included. All sections were scanned using the NanoZoomer 2.0-HT While Slide Imager (Hamamatsu), and image analyses were conducted using the NDP.view2 (v2.7.25). Neurons from the CA4/CA3 regions were evaluated at 20X magnification, while neurons from the temporal cortex were evaluated at $40 \mathrm{X}$ magnification.

\section{Neuropathological assessment}

Hippocampal sections stained with hematoxylin and eosin (H\&E) were scored with regard to gliosis and vacuolation based on a scale from 0 (no astrocytic gliosis/vacuolation) to 3 (severe astrocytic gliosis/vacuolation) as previously reported [5]. Reactive astrocytes were identified from their typically swollen eosinophilic cytoplasm. Only cells with a clearly visible nucleus were evaluated. The neuropathological scoring was performed by two investigators (IHF and GGK) and was done in a region-specific manner resulting in one score from the CA4 region and a separate score from the temporal cortex. In addition, region-specific morphometric analysis of immunoreactivity to $\mathrm{PrP}^{\mathrm{sc}}$ was calculated using Fiji (v.2.0.0) [18]. For the morphometric analyses, single images from the regions of interest were deconvoluted using the color deconvolution tool and an automatic threshold was set using the Isodata threshold mode. Next, all images were binarized and a fraction of measured pixels per image was used to give a score of percentage of $\operatorname{PrP}^{\mathrm{sc}}$ stain per image. The Isodata threshold mode was chosen as it was the best fit for strongly and intermediately stained images. Negative images were not analyzed ( $n=3$ in the CA4 of the MM1 group) due to an overestimation of immunoreactivity. Types of $\operatorname{PrP}^{\mathrm{sc}}$ deposits were not considered in the analyses. However, images from the temporal cortex were acquired in the deeper cortical layers (5 and 6). All images were acquired at 20X magnification. Examples of scores for the neuropathological assessment are shown in Fig. 1.

\section{Statistical analysis}

Statistical analyses were performed in SPSS (v.25.0.0) and Graphpad Prism (v7.0b). Clinical data were compared using t-tests, unless the data were not normally distributed in which case Mann-Whitney U-test was used. Normality was tested using the Shapiro-Wilk test. Equality of variances was tested using Levene's test. A one-way ANOVA 
was conducted between the groups (Ctrl, MM1 and VV2) to test differences of means of the MRC complex analyses. Due to the unequal sample sizes, the Welch's test for unequal variance was used. Eta squared $\left(\eta^{2}\right)$ was used to determine effect size of any significant difference. GamesHowell post hoc test was chosen to decide at what level the groups were significantly different due to their unequal sample sizes and variance. The relationship between neuropathology and MRC deficiency was assessed by pairwise Spearman's correlations. Due to a small sample size, correlations were studied in the entire SCJD group. A linear regression was performed to evaluate covariation between the molecular subtype of SCJD and vacuolation as a marker of neuropathology. A $P$-value $<0.05$ was considered statistically significant in all analyses. Data are presented as mean and $95 \%$ confidence interval (CI).

\section{Results}

Effect of formic acid treatment on immunohistochemistry Using semi-quantification of unstained cytoplasm, we found that FA treatment slightly skewed the measurements towards less MRC deficiency compared to non-FA treated tissue (Fig. 2a). To mitigate the confounder of FA treatment effects on the tissue analyses, we chose instead a visual binary analysis approach to categorize each neuron as either MRC positive or deficient. This approach produced substantially more consistent results between FA treated and untreated tissues (Fig. 2b). Specifically, FA treatment had no effect on the staining of VDAC1 or MRC complexes II, III and V. For the MRC complexes I and IV FA treatment had a minor effect on staining and was associated with a slight underestimation of deficient neurons in FA-treated tissue. Thus, FA treatment could potentially skew our results towards less deficient neurons for complexes I and IV in SCJD. As a result of this, the reported deficiency of MRC complexes I and IV in SCJD may be slightly underestimated in this work.

\section{Neuronal MRC deficiency in SCJD}

To assess the MRC in the sCJD-brain, we performed IHC for MRC complexes I-V in the CA4 region (Fig. 3) and temporal cortex (Fig. 4) of sCJD patients and

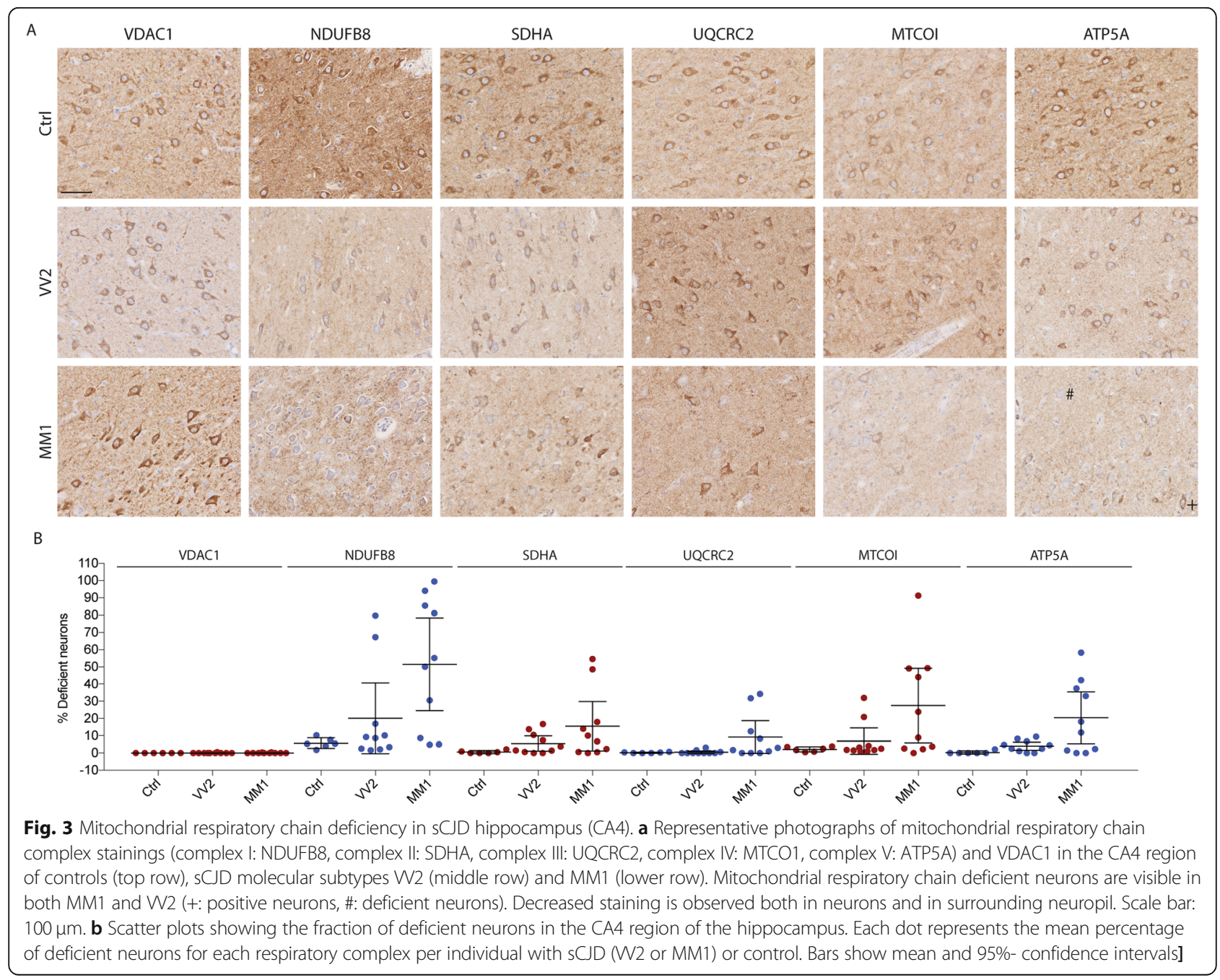




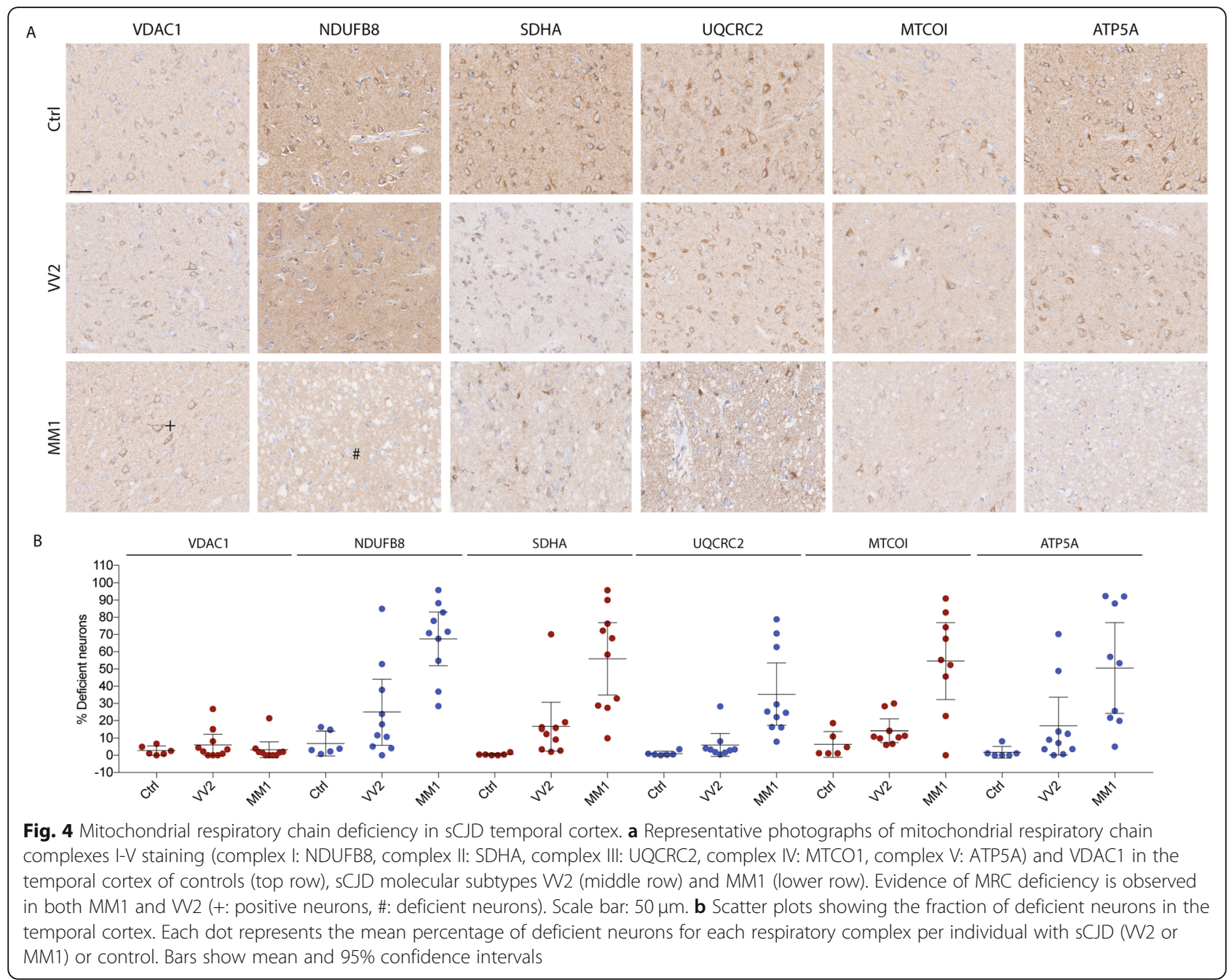

controls. VDAC1 was used as a marker for mitochondrial mass. In the temporal cortex, sCJD patients had a significantly higher fraction of MRC deficient neurons compared to controls. Significant deficiency was found for all MRC complexes (complex I; $P=0.001 \eta^{2}=0.60$, complex II; $P=$ $0.001, \eta^{2}=0.55$, complex III; $P=0.003, \eta^{2}=0.49$, complex IV; $P=0.001, \eta^{2}=0.73$, complex V; $P=0.003, \eta^{2}=0.40$ ). A similar, but overall less pronounced deficiency was found in the CA4/CA3 region (complex I; $P=0.006 \eta^{2}=0.31$, complex II; $P=0.019, \eta^{2}=0.20$, complex III; $P=0.119$, complex IV; $P=0.040, \eta^{2}=0.26$, complex V; $P=0.003$, $\eta^{2}=0.33$; Table 2). VDAC1 staining revealed no difference in mitochondrial mass between groups in either region (temporal cortex; $P=0.542$, CA4/CA3; $P=0.621$ ). The total number of evaluated neurons in each group, means with 95\% CI and results of one way-ANOVA are summarized in Table 2. As reflected in their means and 95\% CI's, there was no difference in staining intensity of neither mitochondrial marker nor MRC complexes between $\mathrm{Ctrl}_{\mathrm{D}}$ and Ctrl. Subject demographics of included individuals are summarised in Table 1.

\section{MRC deficiency is more pronounced in the MM1} molecular subtype of SCJD

Individual assessment of MM1- and VV2 sCJD molecular subtypes showed highly significant differences in terms of MRC deficiency. Compared to controls, tissue from MM1 molecular subtype of SCJD exhibited significant deficiency of all MRC complexes in the temporal cortex (complex I; $P<0.001$, complex II; $P=0.001$, complex III; $P=0.005$, complex IV; $P<0.001$, complex $\mathrm{V} ; P=0.007)$ and of complex I $(P=0.010)$ and complex $\mathrm{V}(P=0.034)$ in the CA4/CA3 region. MRC staining was substantially less affected in VV2 molecular subtype of sCJD. With the exception of mild complex V $(P=0.019)$ deficiency in the CA4/CA3 region, neuronal MRC staining in the VV2 molecular subtype of SCJD was not significantly different from controls (Table 2).

\section{Complex I deficiency in the temporal cortex correlates with sCJD neuropathology}

Next, we sought to investigate whether the MRC deficiency correlated with neuropathology markers of sCJD 
Table 1 Demographic overview, post-mortem delays and fixation times of included samples

\begin{tabular}{|c|c|c|c|c|c|c|}
\hline$\overline{I D}$ & $\mathrm{AOD}$ & Sex & Molecular subtype & DD (months) & PMD (h) & FT (days) \\
\hline \multicolumn{7}{|l|}{ sCJD } \\
\hline sCJD1 & 62 & $\mathrm{~F}$ & W2 & 4 & 72 & 10 \\
\hline sCJD2 & 58 & $\mathrm{~F}$ & W2 & 7 & 24 & 46 \\
\hline sCJD3 & 61 & M & W2 & 3 & 24 & 19 \\
\hline sCJD4 & 73 & $\mathrm{~F}$ & W2 & 4 & 48 & 31 \\
\hline sCJD5 & 69 & M & W2 & 4 & 72 & 22 \\
\hline sCJD6 & 75 & M & W2 & 6 & 24 & 18 \\
\hline sCJD7 & 62 & M & W2 & 2 & 48 & 14 \\
\hline sCJD8 & 65 & M & W2 & 6 & 72 & 21 \\
\hline sCJD9 & 75 & M & W2 & 2 & 24 & 24 \\
\hline sCJD10 & 75 & M & W2 & 8 & 24 & 17 \\
\hline sCJD11 & 80 & $\mathrm{~F}$ & MM1 & 4 & 24 & 27 \\
\hline sCJD12 & 66 & $\mathrm{~F}$ & MM1 & 6 & 96 & 3 \\
\hline sCJD13 & 74 & $\mathrm{~F}$ & MM1 & 4 & 48 & 32 \\
\hline sCJD14 & 68 & $\mathrm{~F}$ & MM1 & 3 & 24 & 44 \\
\hline sCJD15 & 72 & $\mathrm{~F}$ & MM1 & 3 & 24 & 56 \\
\hline sCJD16 & 67 & $M$ & MM1 & 6 & 48 & 112 \\
\hline sCJD17 & 69 & $M$ & MM1 & 2 & 24 & 15 \\
\hline sCJD18 & 72 & $M$ & MM1 & 6 & $<24$ & 16 \\
\hline sCJD19 & 80 & $\mathrm{~F}$ & MM1 & 1.5 & 48 & 98 \\
\hline sCJD20 & 70 & $M$ & MM1 & 8 & 24 & 30 \\
\hline \multicolumn{7}{|l|}{$\left.C \operatorname{trl}\right|_{D}$} \\
\hline Ctrl1 & 67 & $\mathrm{~F}$ & - & - & 24 & 31 \\
\hline Ctrl2 & 78 & $\mathrm{~F}$ & - & - & 48 & 14 \\
\hline Ctrl3 & 81 & $\mathrm{~F}$ & - & - & 24 & 37 \\
\hline \multicolumn{7}{|l|}{ Ctrl } \\
\hline Ctrl 4 & 65 & $\mathrm{~F}$ & - & - & 24 & 20 \\
\hline Ctrl5 & 73 & $\mathrm{~F}$ & - & - & 48 & 22 \\
\hline Ctrl6 & 81 & $M$ & - & - & 24 & 15 \\
\hline
\end{tabular}

CJD Sporadic Creutzfeldt-Jakob disease, $C t r l_{D}$ Disease-control, Ctrl Control, AOD Age of death, PMD Post-mortem delay (hours), $F T$ Fixation time (days), $F$ Female, $M$ Male, $D D$ Disease duration in months

(Fig. 5). To this end, we chose complex I deficiency as a representative marker of MRC deficiency. Individual scores of neuropathology markers and means with $95 \%$ $\mathrm{CI}$ are listed in Table 3. As expected, vacuolation and $\mathrm{PrP}^{\mathrm{sc}}$-staining correlated significantly with gliosis in all examined areas (Fig. 5a). Intriguingly, in the temporal cortex, we found a significant positive correlation between the amount of complex I deficient neurons and vacuolation $\left(P<0.001, \mathrm{r}_{\mathrm{s}}=0.725\right)$, gliosis $\left(P<0.001, \mathrm{r}_{\mathrm{s}}=\right.$ $0.771)$ and $\operatorname{PrP}^{\mathrm{sc}}$ stain $\left(P=0.018, \mathrm{r}_{\mathrm{s}}=0.537\right.$; Fig. $\left.5 \mathrm{~b}\right)$. In contrast, the hippocampal regions CA4/CA3 showed no correlation with any of the neuropathology markers (vacuolation; $P=0.699$, gliosis; $P=0.605, \operatorname{Pr}^{\mathrm{sC}}$ stain; $P=$ 0.109; Fig. 5c). Disease duration did not correlate with the percentage of complex I deficient neurons in either temporal cortex $(P=0.227)$ or the CA4/CA3 hippocampal regions $(P=0.951)$. Linear regression with vacuolation as covariate confirmed that molecular subtype of $\mathrm{sCJD}$ is a predictor of MRC deficiency (Beta $=0.40, P=$ 0.019), however, this difference could be partly confounded by severity of vacuolation (Beta $=0.57, P=$ 0.002).

\section{Discussion}

We show that neuronal MRC deficiency affecting all respiratory complexes occurs in the brain of patients with sCJD. This deficiency shows a strong association with the severity of pathological markers of disease and has a predilection for the MM1 molecular disease subtype. In spite of the pronounced MRC loss, the outer mitochondrial 
Table 2 Visual analyses of mitochondrial respiratory chain staining

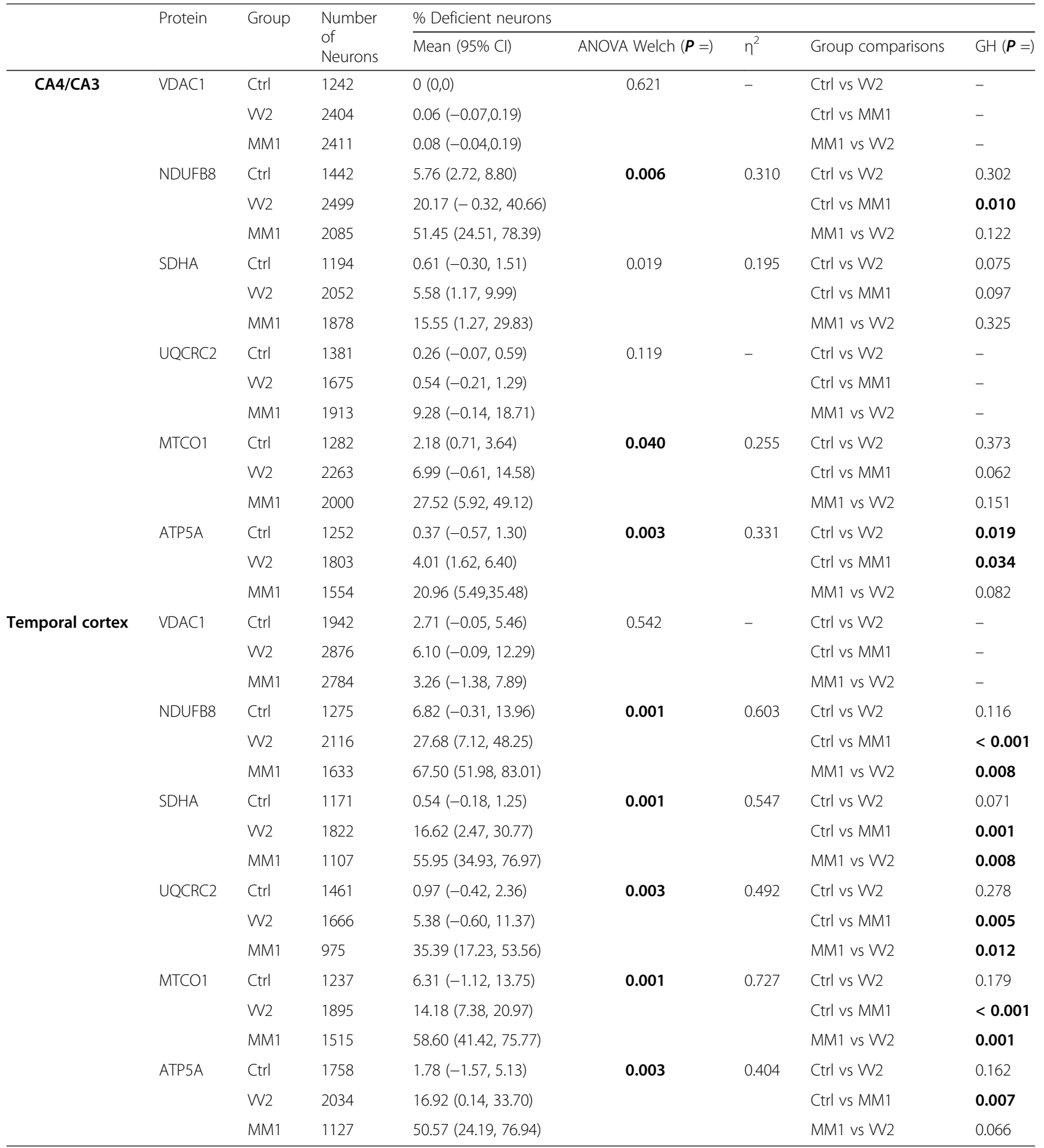

The table summarizes results of one-way ANOVA and eta squared analysis of effect where a significant difference at the group level. $95 \%$ Cl: $95 \%$ confidence interval, $\eta^{2}$; eta squared, GH: Games-Howell, Ctrl: control, MM1: MM1 molecular subtype of SCJD, VV2; VV2 molecular subtype of SCJD. Statistically significant $P$ values are in bold type

membrane marker VDAC1 remains intact, indicating that the total neuronal mitochondrial mass remains unchanged. The finding of deficient MRC in SCJD corroborate the work of Ansoleaga et al., who reported deficiency of multiple MRC complexes in the frontal cortex of MM1 type sCJD [11].

Neuronal MRC deficiency in SCJD shows a strong correlation with the severity of the neurodegenerative 

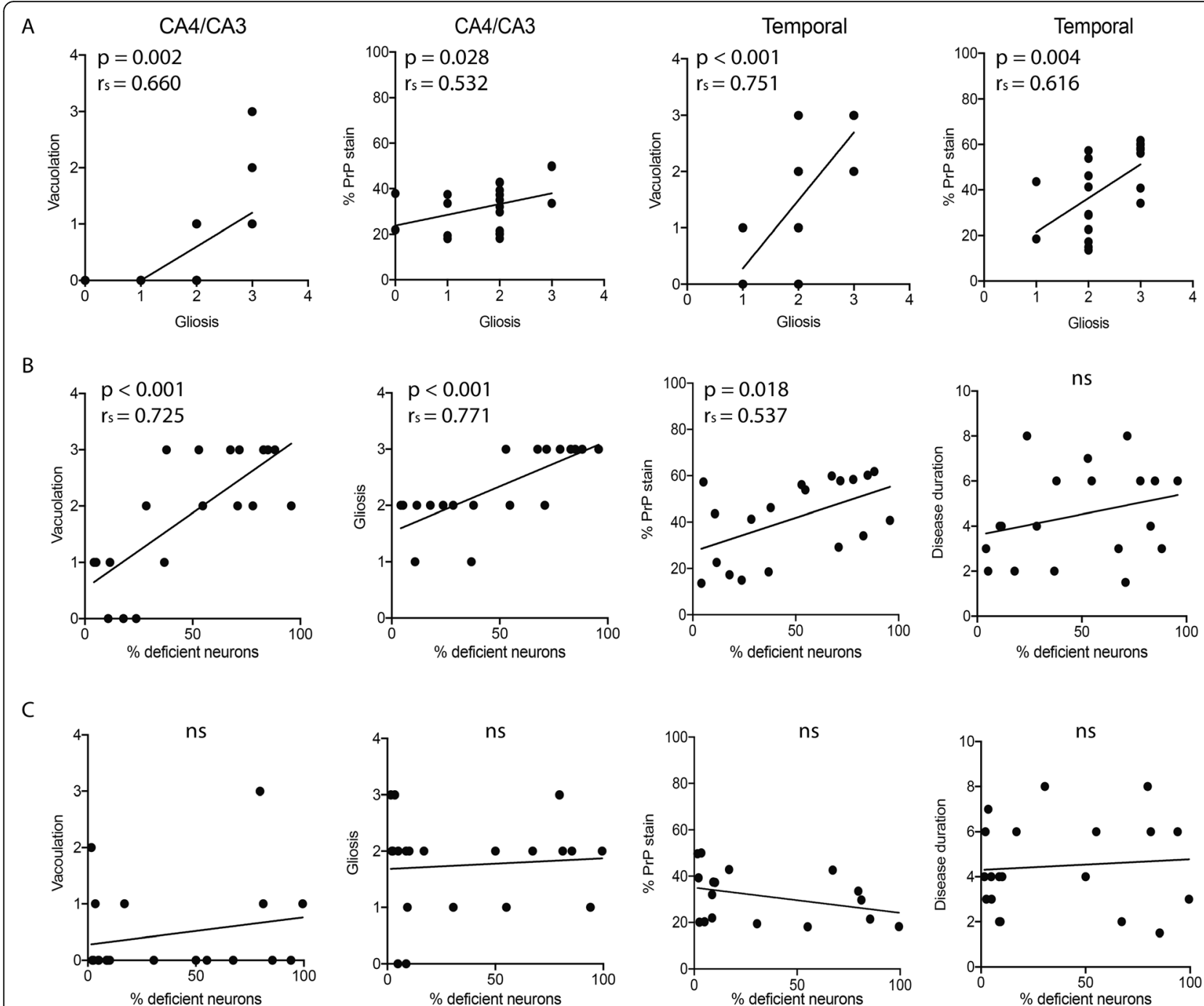

Fig. 5 Neuropathology correlates with complex I deficiency in the temporal cortex. Correlation plots of clinicopathological variables and MRC deficiency, exemplified by complex I (NDUFB8). Vacuolation and PrPsc-immunoreactivity correlate with gliosis in CA4/CA3 and temporal cortex (a). Vacuolation, gliosis and PrPsc-immunoreactivity correlate with mitochondrial complex I deficiency in the temporal cortex, (b) but not the hippocampal regions of CA4/CA3 (c)

changes. It has a clear regional predilection for the severely affected temporal cortex, whereas the relatively spared CA4/CA3 hippocampal regions show only a mild decrease in MRC staining. Moreover, the extent of MRC deficiency in the temporal cortex shows a positive correlation with the severity of neuropathological changes including gliosis, vacuolation and $\operatorname{PrP}^{\mathrm{sc}}$ load. We believe that the lack of association between MRC deficiency and severity of neuropathology in the CA4/CA3 region may reflect insufficient statistical power due to the substantially milder MRC involvement in that area.

The severity of MRC deficiency is associated with the molecular subtype of sCJD. MRC deficiency is significantly more pronounced in neurons of individuals with MM1 type
sCJD compared to VV2 type sCJD. In fact, with the exception of complex V, MRC deficiency in VV2 type sCJD was not statistically significant compared to controls, although a trend was seen for higher numbers of MRC-deficient neurons. Regression analysis shows that the difference between molecular subtype of SCJD is partly confounded by the more severe pathological changes in MM1 molecular phenotype. Spongiform changes are more pronounced in the neocortex of MM1 type SCJD compared to VV2, where the basal ganglia and thalamus are predominantly affected [19]. However, as disease subtype remains a significant predictor of MRC deficiency in our model, we cannot exclude the possibility of additional factors rendering MM1 neurons more susceptible to MRC impairment. 
Table 3 Neuropathological assessments

\begin{tabular}{|c|c|c|c|c|c|c|c|}
\hline \multirow[b]{2}{*}{ ID } & \multirow[b]{2}{*}{ Type } & \multicolumn{3}{|l|}{ CA4/CA3 } & \multicolumn{3}{|l|}{ Temporal } \\
\hline & & $\%$ PrP stain & Gliosis & Vacuo & $\%$ PrP stain & Gliosis & Vacuo \\
\hline \multicolumn{8}{|l|}{ sCJD } \\
\hline sCJD1 & W2 & 37.26 & 2 & 0 & 22.64 & 2 & 1 \\
\hline sCJD2 & W2 & 50.04 & 3 & 1 & 56.09 & 3 & 3 \\
\hline sCJD3 & W2 & 20.15 & 2 & 0 & 13.65 & 2 & 1 \\
\hline sCJD4 & W2 & 49.70 & 3 & 2 & 28.90 & 2 & 2 \\
\hline sCJD5 & W2 & 32.07 & 2 & 0 & 43.63 & 1 & 0 \\
\hline sCJD6 & W2 & 39.31 & 2 & 0 & 46.17 & 2 & 3 \\
\hline sCJD7 & W2 & 42.61 & 2 & 0 & 17.31 & 2 & 0 \\
\hline sCJD8 & W2 & 42.88 & 2 & 1 & 60.23 & 3 & 3 \\
\hline sCJD9 & W2 & 37.42 & 1 & 0 & 57.27 & 2 & 1 \\
\hline sCJD10 & W2 & 33.55 & 3 & 3 & 14.93 & 2 & 0 \\
\hline Mean $(95 \% \mathrm{Cl})$ & & $38.50(32.19,44.81)$ & $2.20(1.75,2.65)$ & $0.7(-0.06,1.46)$ & $36.08(22.75,49.41)$ & $2.10(1.69,2.51)$ & $1.4(0.50,2.30)$ \\
\hline sCJD11 & MM1 & - & 2 & 0 & 34.13 & 3 & 3 \\
\hline sCJD12 & MM1 & 18.16 & 1 & 0 & 53.85 & 2 & 2 \\
\hline sCJD13 & MM1 & - & 0 & 0 & 41.26 & 2 & 2 \\
\hline sCJD14 & MM1 & 18.17 & 2 & 1 & 61.81 & 3 & 3 \\
\hline sCJD15 & MM1 & 20.25 & 2 & 0 & 59.89 & 3 & 3 \\
\hline sCJD16 & MM1 & - & 1 & 0 & 40.80 & 3 & 2 \\
\hline sCJD17 & MM1 & 21.99 & 0 & 0 & 18.50 & 1 & 1 \\
\hline sCJD18 & MM1 & 29.68 & 2 & 1 & 58.40 & 3 & 2 \\
\hline sCJD19 & MM1 & 21.48 & 2 & 0 & 29.17 & 2 & 2 \\
\hline sCJD20 & MM1 & 19.43 & 1 & 0 & 57.80 & 3 & 3 \\
\hline Mean $(95 \% \mathrm{Cl})$ & & $21.31(17.63,24.99)$ & $1.43(0.70,2.16)$ & $0.29(-0.17,0.74)$ & $48.49(32.50,64.48)$ & $2.43(1.70,3.16)$ & $2.29(1.59,2.98)$ \\
\hline \multicolumn{8}{|l|}{$\mathrm{Ctrl}_{\mathrm{D}}$} \\
\hline Ctrl1 & - & - & 1 & 0 & - & 1 & 0 \\
\hline Ctrl2 & - & - & 1 & 0 & - & 0 & 0 \\
\hline Ctrl3 & - & - & 0 & 0 & - & 0 & 0 \\
\hline \multicolumn{8}{|l|}{ Ctrl } \\
\hline Ctrl4 & - & - & 0 & 0 & - & 0 & 0 \\
\hline Ctrl5 & - & - & 0 & 0 & - & 1 & 0 \\
\hline Ctrl6 & - & - & 2 & 0 & - & 1 & 0 \\
\hline
\end{tabular}

sCJD = sporadic Creutzfeldt-Jakob disease, Ctrl D Diseased control, Ctrl Control, MM1 MM1 molecular subtype sCJD, VV2 VV2 molecular subtype sCJD, \% PrP stain $=\%$ morphometric score of prion stain, Gliosis $=$ score gliosis, Vacuo $=$ score vacuolation

Our findings suggest that MRC deficiency is an integral part of sCJD pathology and plays an important role in the pathogenesis of the disorder. While MRC deficiency correlates strongly with the severity of disease specific pathological markers, the fact that it is also present in morphologically normal neurons of the mildly affected CA4/CA3 region suggests it is not a terminal phenomenon in dying neuronal populations. The mechanisms underlying MRC loss in SCJD remain unknown. Since mitochondrial mass is unchanged, it is possible MRC is depleted via an active downregulation or increased degradation of the peptide subunits of the respiratory complexes. mtDNA encodes subunits of complexes I, III, IV and V and quantitative and/or qualitative mtDNA defects are a common cause of MRC deficiency [20]. However, mtDNA defects should not affect complex II, which is entirely encoded in the nucleus, and very rarely cause a uniform loss of the remaining respiratory complexes. Therefore, the MRC deficiency of sCJD does not fit the profile of pure mtDNA defects.

The observed pan-respiratory complex deficiency without a change in the mitochondrial outer membrane marker VDAC1 may reflect damage to the inner mitochondrial membrane. This is in line with previous 
reports of abnormal mitochondrial cristae architecture in prion infected rodents $[8,21,22]$. Interestingly, while $\operatorname{PrP}^{\mathrm{c}}$ has been shown to localize mostly on the plasma membrane, it has been suggested that it may also be present in the inner mitochondrial membrane of healthy mice [23]. Furthermore, dysmorphic mitochondria with abnormal cristae morphology have been observed in PRNP knockout mice [24]. It is therefore possible that $\operatorname{PrP}^{\mathrm{sc}}$ aggregation injures the inner mitochondrial membrane, both directly and via loss of its physiological counterpart, $\operatorname{PrP}^{\mathrm{c}}$.

Irrespective of the cause, deficiency of the entire MRC is expected to compromise neuronal metabolism causing ATP depletion and a shift toward increased glycolysis [25]. Neurons are highly dependent on oxidative phosphorylation and use most of their ATP to maintain their membrane potential. This is achieved via the action of the sodium-potassium ATPase, which maintains the intraand extracellular concentrations of sodium and potassium ions against their electrochemical gradients and, by extension, regulates intracellular water balance [26]. Pump failure due to ATP deficiency causes a shift of water from the extracellular to the intracellular compartment (i.e. cytotoxic edema), leading to disruption of essential cellular processes and ultimately cell death. In fact, diffusion weighted imaging (DWI) of the brain typically shows evidence of restricted water diffusion in affected areas of the sCJD brain [27], suggesting that cytotoxic edema is indeed an important mechanism underlying neuronal death in sCJD. Based on our findings, we propose that cytotoxic changes in the SCJD brain are caused by ATP depletion due to neuronal MRC deficiency. Similar signs of restricted water diffusion on MRI are seen in other disorders involving neuronal energy failure such as mitochondrial disease [28-30], hypoxia/ischemia [31], hypoglycaemia $[32,33]$ and carbon monoxide intoxication [34].

Another consequence of neuronal MRC impairment in sCJD would be a shift toward glycolytic metabolism, resulting in higher production of lactate. Elevated concentration of cerebrospinal fluid (CSF) lactate has indeed been reported in patients with CJD, corroborating this hypothesis [35]. In addition to decreased ATP production, a glycolytic shift could render neurons more susceptible to oxidative damage. Normally, neurons can consume glucose through the pentose phosphate pathway, which helps to regenerate reduced glutathione, an important component against oxidative stress. Upregulation of glycolysis may therefore cause increased levels of oxidized glutathione, in turn, increased susceptibility to the formation of reactive oxygen species in neurons [36]. Oxidative damage has indeed been implicated in cell models of prion disease [37-39].

While cause and effect cannot be confidently discerned by this type of study, our findings strongly suggest that MRC loss in SCJD is deleterious and actively involved in the neurodegenerative process. Interestingly, the pattern of MRC deficiency in SCJD is distinct from that of other neurodegenerative proteinopathies. In Parkinson's disease, MRC deficiency is selective for complex I (and to a much lesser degree complex IV) and does not correlate with the severity of neurodegeneration. In fact, unlike sCJD where the load of $\operatorname{PrP}^{\text {sc }}$ correlates with MRC deficiency, in PD there is an inverse relationship between complex I loss and Lewy pathology leading to the hypothesis that it may be a partly protective event $[17,40]$. It is conceivable that downregulation of complex I levels without a decrease in the remaining respiratory complexes, as observed in PD, serves to limit reactive oxygen species (ROS) production and oxidative damage without a major compromise in energy transduction. In contrast, the global MRC loss seen in sCJD is highly likely to cause ATP depletion.

In conclusion, we show that mitochondrial dysfunction is an important mechanism underlying neuronal injury and neurodegeneration in SCJD. These findings provide an explanation for the clinical observations of restricted MRI diffusion and elevated CSF lactate in patients. Our findings suggest that mitochondria should be the focus of further study in SCJD and should be assessed as potential therapeutic targets for this incurable and devastating disorder.

\section{Abbreviations \\ sCJD: Sporadic creutzfeldt-jakob disease; PrPsc: Disease associated prion protein; $\operatorname{PrP}^{c}$ : Naturally occurring prion protein; MRC: Mitochondrial respiratory chain; mtDNA: Mitochondrial DNA; PrPC: Prion protein; FA: Formic acid; Ctrl: Control; Ctrl D: Disease-control; CJD: Creutzfeldt-Jakob disease; MRI: Magnetic resonance imaging; IHC: Immunohistochemistry; HRP: Horseradish peroxidase; DAB: 3,3'-Diaminobenzidine; Cl: Confidence interval; DWI: Diffusion weighted imaging; CSF: Cerebrospinal fluid; ROS: Reactive oxygen species}

\section{Acknowledgements}

Not applicable.

\section{Authors' contributions}

Conception, design and funding: IHF, GGK and CT Acquisition and analysis of data: IHF, GR, SK, AL, TS. Drafting the manuscript: IHF, GGK, CD, CT. Statistical analysis: IHF. Critical revision of the manuscript: GR, SK, AL, TS. The author(s) read and approved the final manuscript.

\section{Funding}

This work was supported by grants from the Regional Health Authority of Western Norway (grant no 911903 and 911988), the Research Council of Norway (grant no ES633272), and Bergen Research Foundation (BFS2017REK05)

\section{Availability of data and materials}

All data used and/or analysed during the current study available from the corresponding author on reasonable request.

\section{Ethics approval and consent to participate}

Samples were collected following local regulations and the study was performed in the frame of a study ("Molecular neuropathologic investigation of neurodegenerative diseases") approved by the Ethical Committee of the Medical University of Vienna (Nr. 396/2011). All experiments were performed in accordance with relevant Austrian guidelines and regulations. In addition, 
the work presented here was approved by the Regional Committee for Medical and Health Research Ethics, Western Norway (REK 2018/1666). The manuscript does not contain information or images that could lead to an identification of the individual or which could violate any personal rights.

\section{Consent for publication}

Not applicable.

\section{Competing interests}

The authors declare that they have no competing financial interests.

\section{Author details}

${ }^{1}$ Neuro-SysMed, Department of Neurology, Haukeland University Hospital, 5021 Bergen, Norway. ${ }^{2}$ Department of Clinical Medicine, University of Bergen, $\mathrm{Pb} 7804,5020$ Bergen, Norway. ${ }^{3}$ Institute of Neurology, Medical University of Vienna, Vienna, Austria. ${ }^{4}$ Tanz Centre for Research in Neurodegenerative Disease, University of Toronto, Toronto, Ontario, Canada. ${ }^{5}$ Laboratory Medicine Program, University Health Network, Toronto, Canada.

\section{Received: 3 January 2020 Accepted: 13 March 2020}

\section{Published online: 16 April 2020}

\section{References}

1. Prusiner SB (2001) Shattuck lecture--neurodegenerative diseases and prions. N Engl J Med 344:1516-1526. https://doi.org/10.1056/ NEJM200105173442006

2. Ironside JW, Ritchie DL, Head MW (2017) Prion diseases. Handb Clin Neurol 145:393-403. https://doi.org/10.1016/B978-0-12-802395-2.00028-6

3. Parchi P, Castellani R, Capellari S et al (1996) Molecular basis of phenotypic variability in sporadic Creutzfeldt-Jakob disease. Ann Neurol 39:767-778. https://doi.org/10.1002/ana.410390613

4. Parchi P, Strammiello R, Giese A et al (2011) Phenotypic variability of sporadic human prion disease and its molecular basis: past, present, and future. Acta Neuropathol 121:91-112. https://doi.org/10.1007/s00401-010-0779-6

5. Parchi P, Giese A, Capellari S et al (1999) Classification of sporadic Creutzfeldt-Jakob disease based on molecular and phenotypic analysis of 300 subjects. Ann Neurol 46:224-233

6. Lin MT, Beal MF (2006) Mitochondrial dysfunction and oxidative stress in neurodegenerative diseases. Nature 443:787-795. https://doi.org/10.1038/ nature05292

7. Aiken JM, Williamson JL, Marsh RF (1989) Evidence of mitochondrial involvement in scrapie infection. J Virol 63:1686-1694

8. Siskova Z, Mahad DJ, Pudney C et al (2010) Morphological and functional abnormalities in mitochondria associated with synaptic degeneration in prion disease. Am J Pathol 177:1411-1421. https://doi.org/10.2353/ajpath. 2010.091037

9. Faris R, Moore RA, Ward A et al (2017) Mitochondrial respiration is impaired during late-stage hamster prion infection. J Virol 91. https://doi.org/10.1128/ JVI.00524-17

10. Yuan F, Yang L, Zhang Z et al (2013) Cellular prion protein (PrPC) of the neuron cell transformed to a PK-resistant protein under oxidative stress, comprising main mitochondrial damage in prion diseases. J Mol Neurosci 51:219-224. https://doi.org/10.1007/s12031-013-0008-6

11. Ansoleaga B, Garcia-Esparcia P, Llorens F et al (2016) Altered Mitochondria, Protein Synthesis Machinery, and Purine Metabolism Are Molecular Contributors to the Pathogenesis of Creutzfeldt-Jakob Disease. J Neuropathol Exp Neurol Published Online First. https://doi.org/10.1093/ jnen/nlw048

12. Tzoulis C, Tran GT, Coxhead J et al (2014) Molecular pathogenesis of polymerase $\gamma$-related neurodegeneration. Ann Neurol 76:66-81. https://doi. org/10.1002/ana.24185

13. Nido GS, Dick F, Toker L, et al. Common gene expression signatures in Parkinson's disease are driven by changes in cell composition. BioRxiv. 2019. https://doi.org/10.1101/778910.

14. Kaneko M, Sugiyama N, Sasayama D et al (2008) Prion disease causes less severe lesions in human hippocampus than other parts of brain Psychiatry Clin Neurosci 62:264-270. https://doi.org/10.1111/j.1440-1819. 2008.01792.x

15. Kovacs GG Neuropathology of Neurodegenerative Diseases: A practical Guide. Cambridge University Press, Cambridge, pp 55-69
16. Parchi P, de Boni L, Saverioni D et al (2012) Consensus classification of human prion disease histotypes allows reliable identification of molecular subtypes: an inter-rater study among surveillance centres in Europe and USA. Acta Neuropathol 124:517-529. https://doi.org/10.1007/s00401-0121002-8

17. Flones $\mathbb{H}$, Fernandez-Vizarra $E$, Lykouri M et al (2017) Neuronal complex I deficiency occurs throughout the Parkinson's disease brain, but is not associated with neurodegeneration or mitochondrial DNA damage. Acta Neuropathol Published Online First. https://doi.org/10.1007/s00401-017-1794-7

18. Schindelin J, Arganda-Carreras I, Frise E et al (2012) Fiji: an open-source platform for biological-image analysis. Nat Methods 9:676-682. https://doi org/10.1038/nmeth.2019

19. Kovacs GG, Head MW, Bunn T et al (2000) Clinicopathological phenotype of codon 129 valine homozygote sporadic Creutzfeldt-Jakob disease. Neuropathol Appl Neurobiol 26:463-472. https://doi.org/10.1046/j.13652990.2000.00279.x

20. Berg JM; T. Oxidative Phosphorylation. In: Berg JM; T, ed. Biochemistry. United States of America: : W. H. Freeman and Company 2007. 502-40

21. Lee DW, Sohn HO, Lim HB et al (1999) Alteration of free radical metabolism in the brain of mice infected with scrapie agent. Free Radic Res 30:499-507. https://doi.org/10.1080/10715769900300541

22. Choi SI, Ju WK, Choi EK et al (1998) Mitochondrial dysfunction induced by oxidative stress in the brains of hamsters infected with the $263 \mathrm{~K}$ scrapie agent. Acta Neuropathol 96:279-286. https://doi.org/10.1007/s004010050895

23. Sisková Z, Mahad DJ, Pudney C et al (2010) Morphological and functional abnormalities in mitochondria associated with synaptic degeneration in prion disease. Am J Pathol 177:1411-1421. https://doi.org/10.2353/ajpath. 2010.091037

24. Faris R, Moore RA, Ward A et al (2017) Cellular prion protein is present in mitochondria of healthy mice. Sci Rep 7:41556. https://doi.org/10.1038/srep41556

25. Miele G, Jeffrey M, Turnbull D et al (2002) Ablation of cellular prion protein expression affects mitochondrial numbers and morphology. Biochem Biophys Res Commun 291:372-377. https://doi.org/10.1006/bbrc.2002.6460

26. Ozawa S, Ueda S, Imamura H et al (2015) Glycolysis, but not mitochondria, responsible for intracellular ATP distribution in cortical area of podocytes. Sci Rep 5:18575, https://doi.org/10.1038/srep18575

27. Gerkau NJ, Rakers C, Petzold GC et al (2017) Differential effects of energy deprivation on intracellular sodium homeostasis in neurons and astrocytes. J Neurosci Res 95:2275-2285. https://doi.org/10.1002/jnr.23995

28. Rudge $\mathrm{P}$, Hyare $\mathrm{H}$, Green A et al (2018) Imaging and CSF analyses effectively distinguish CJD from its mimics. J Neurol Neurosurg Psychiatry 89:461-466. https://doi.org/10.1136/jnnp-2017-316853

29. Tzoulis C, Bindoff LA (2009) Serial diffusion imaging in a case of mitochondrial encephalomyopathy, lactic acidosis, and stroke-like episodes. Stroke 40:e15-e17. https://doi.org/10.1161/STROKEAHA.108.523118

30. Tzoulis C, Neckelmann G, Mork SJ et al. (2010) Localized cerebral energy failure in DNA polymerase gamma-associated encephalopathy syndromes. Brain 133:1428-37. https://doi.org/10.1093/brain/awq067

31. Schaefer PW, Grant PE, Gonzalez RG (2000) Diffusion-weighted MR imaging of the brain. Radiology 217:331-345. https:/doi.org/10.1148/radiology.217.2.ro0nv24331

32. Yoneda Y, Yamamoto S (2005) Cerebral cortical laminar necrosis on diffusion-weighted MRI in hypoglycaemic encephalopathy. Diabet Med 22: 1098-1100. https://doi.org/10.1111/j.1464-5491.2005.01568.x

33. Bottcher J, Kunze A, Kurrat C et al (2005) Localized reversible reduction of apparent diffusion coefficient in transient hypoglycemia-induced hemiparesis. Stroke 36:e20-e22. https://doi.org/10.1161/01.STR.0000155733.65215.c2

34. Kondo A, Saito Y, Seki A et al (2007) Delayed neuropsychiatric syndrome in a child following carbon monoxide poisoning. Brain and Development 29: 174-177. https://doi.org/10.1016/j.braindev.2006.08.002

35. Awerbuch G, Peterson P, Sandyk R (1988) Elevated cerebrospinal fluid lactic acid levels in Creutzfeldt-Jakob disease. Int J Neurosci 42:1-5

36. Herrero-Mendez A, Almeida A, Fernandez E et al (2009) The bioenergetic and antioxidant status of neurons is controlled by continuous degradation of a key glycolytic enzyme by APC/C-Cdh1. Nat Cell Biol 11:747-752. https:// doi.org/10.1038/ncb188

37. Pamplona R, Naudi A, Gavin R et al (2008) Increased oxidation, glycoxidation, and lipoxidation of brain proteins in prion disease. Free Radic Biol Med 45:1159-1166, https://doi.org/10.1016/jfreeradbiomed.2008.07.009

38. Sorce S, Nuvolone M, Keller A et al (2014) The role of the NADPH oxidase NOX2 in prion pathogenesis. PLoS Pathog 10:e1004531. https://doi.org/10. 1371/journal.ppat.1004531 
39. Herrmann US, Sonati T, Falsig J et al (2015) Prion infections and anti-PrP antibodies trigger converging neurotoxic pathways. PLoS Pathog 11: e1004662. https://doi.org/10.1371/journal.ppat.1004662

40. Reeve AK, Park T-K, Jaros E et al (2012) Relationship between mitochondria and a-synuclein: a study of single substantia nigra neurons. Arch Neurol 69: 385-393. https://doi.org/10.1001/archneurol.2011.2675

\section{Publisher's Note}

Springer Nature remains neutral with regard to jurisdictional claims in published maps and institutional affiliations.

Ready to submit your research? Choose BMC and benefit from:

- fast, convenient online submission

- thorough peer review by experienced researchers in your field

- rapid publication on acceptance

- support for research data, including large and complex data types

- gold Open Access which fosters wider collaboration and increased citations

- maximum visibility for your research: over $100 \mathrm{M}$ website views per year

At BMC, research is always in progress. 